\title{
Estrutura Interna da Escala Cognitiva de Ansiedade (ECOGA)
}

\author{
Makilim Nunes Baptista ${ }^{1}$ \\ Eliane Mary de Oliveira Falcone ${ }^{2}$ \\ Monique Placido Viegas ${ }^{2}$ \\ Evlyn Rodrigues Oliveira ${ }^{2}$ \\ Stèphanie Krieger ${ }^{3}$ \\ Luisa Braga Pereira' \\ Ana Júlia de Carvalho Pereira Alves² \\ Marcelo Leonel Peluso ${ }^{2}$ \\ Filipe Tomé da Silva \\ ${ }^{1}$ Universidade São Francisco, Campinas, São Paulo, Brasil \\ ${ }^{2}$ Universidade do Estado do Rio de Janeiro, Rio de Janeiro, Rio de Janeiro, Brasil \\ ${ }^{3}$ Universidade Estácio de Sá, Rio de Janeiro, Rio de Janeiro, Brasil
}

\section{Resumo}

Este estudo objetivou investigar evidências de validade de estrutura interna para a Escala Cognitiva de Ansiedade (ECOGA) por análise fatorial exploratória (AFE). A amostra foi formada por 874 participantes, sendo 656 da população geral, 179 de instituições de saúde e 39 de consultórios particulares com hipótese diagnóstica de transtornos de ansiedade. A maioria da amostra foi masculina (55\%), solteiros $(61,1 \%)$ com ensino superior incompleto (46\%). A análise paralela da ECOGA, contendo inicialmente 73 itens, demonstrou a existência de até quatro fatores, a MAP com três e a Hull com um. Análises com três e quatro fatores (teoricamente aceitáveis) foram realizadas, utilizando cargas fatoriais acima de 0,40 e 0,50 . O modelo teórico e psicométrico mais adequado compreendeu 31 itens e três fatores, com cargas fatoriais mínimas de 0,50. Os índices de ajuste (CFI, GFI, RMSEA) unidimensionalidade (ÚNICO e MIREAL) e confiabilidade (Orion e Cronbach) complementaram as adequadas propriedades psicométricas desta versão.

Palavras-chave: distúrbios da ansiedade, avaliação psicológica, testes psicológicos, análise fatorial

\section{Internal Structure of the Cognitive Anxiety Scale (ECOGA)}

\begin{abstract}
This study aimed to investigate evidence of internal structure validity for the Anxiety Cognitive Scale (ECOGA) by Exploratory Factor Analysis (EFA). The sample consisted of 874 participants, 656 of the general population, 179 of health institutions and 39 of private practices with a diagnostic hypothesis of anxiety disorders. Men comprised $55 \%$ of the total sample, which was also mostly single (61.1\%) and with incomplete higher education (46\%). The parallel analysis of this scale, initially containing 73 items, showed the existence of up to 4 factores, the MAP with 3 and the Hull with 1 grouping of items. Analyses with 3 and 4 factors (theoretically acceptable) were performed, using factor loads above 0.40 and 0.50 . The most appropriate theoretical and psychometric model comprised 31 items and 3 factors, with a minimum factor load of 0.50. Indexes of fit (CFI, GFI, RMSEA), unidimensionality (UNICO, MIREAL) and reliability (Orion, Cronbach) complemented the appropriate psychometric properties of this version.

Keywords: anxiety disorders; psychological assessment; psychological testing; factor analysis.
\end{abstract}

Estructura Interna de la Escala Cognitiva de Ansiedad (ECOGA)

\section{Resumen}

Este estudio tuvo por objetivo investigar evidencias de validez de la estructura interna para la Escala Cognitiva de Ansiedad (ECOGA) por el análisis factorial exploratorio. La muestra fue formada por 874 participantes, 656 de población general, 179 de instituciones de salud y 39 de consultorios privados con hipótesis diagnóstica de trastornos de ansiedad. La mayoría de la muestra fue masculina $(55 \%)$, solteros $(61,1 \%)$ con enseñanza superior incompleta $(46 \%)$. El análisis paralelo de la escala, conteniendo inicialmente 73 ítems, demostró la existencia de hasta cuatro factores, la MAP con tres y la Hull, uno. Se realizaron análisis con tres y cuatro factores (teóricamente aceptables), utilizando cargas factoriales superior a 0,40 y 0,50 . El modelo teórico y psicométrico más apropiado comprendió 31 ítems y tres factores, con cargas factoriales mínimas de 0,50. Los índices de ajuste (CFI, GFI, RMSEA), unidimensionalidad (UNICO, MIREAL) y confiabilidad (Orion, Cronbach) complementaron las adecuadas propiedades psicométricas de esta versión.

Palabras clave: trastornos de la ansiedad; evaluación psicológica; testes psicológicos; análisis factorial. 
A ansiedade e seus transtornos estão entre as maiores causas de incapacitação em todo o mundo, conforme sugere a Global Burden of Disease (2017). A prevalência estimada dos transtornos de ansiedade ao longo da vida é de $28,8 \%$. Além disso, a ansiedade clínica é crescente no mundo, gerando prejuízos, como redução da qualidade de vida, do funcionamento social e ocupacional (Clark \& Beck, 2012). Dados brasileiros indicam uma prevalência de 12 meses de 12,7\% (Blay et al., 2018).

A avaliação cognitiva distorcida acerca do perigo tem sido apontada como um elemento central da ansiedade, constituindo um importante fator de vulnerabilidade para a compreensão da etiologia, manutenção e tratamento dos transtornos emocionais (Payne, Ellard, Farchione, Fairholme, \& Barlow, 2016). Indivíduos ansiosos tendem a superestimar o perigo e a sua probabilidade de ocorrência, além de se considerarem incapazes de enfrentar situações percebidas como ameaçadoras (Barlow \& Durand, 2017; Clark \& Beck, 2012). Eles percebem a própria emoção como incontrolável e insuportável, são intolerantes a incertezas e aflições, usam estratégias ineficazes de regulação da emoção, por meio da evitação, o que contribui para a manutenção da ansiedade (Payne et al., 2016).

A presença de padrões cognitivos distorcidos compartilhados em diferentes transtornos emocionais tem sido amplamente reconhecida na literatura (Drost, van der Does, van Hemert, Penninx, \& Spinhoven, 2014; Sydenham, Beardwood, \& Rimes, 2017). Elevadas taxas de comorbidade entre os transtornos de ansiedade também indicam a existência de mais aspectos comuns entre eles do que diferenças, significando que alguns transtornos podem ser considerados como variações de uma mesma síndrome (Franzin, Caetano, \& Melo, 2018). Assim, além do transtorno de ansiedade principal, a presença de outro transtorno de ansiedade, bem como a depressão, tem sido identificada em cerca de 60\% dos pacientes diagnosticados (Payne et al., 2016). Com base em ampla revisão de estudos sobre o tema, Payne, Ellard, Farchione, Fairholme e Barlow (2016) sugerem que a avaliação e o tratamento dos transtornos emocionais devem se basear em uma abordagem transdiagnóstica mais unificada, pautadas em estudos recentes sobre a natureza da psicopatologia e das ciências das emoções, em alternativa às categorias diagnósticas específicas de cada transtorno de ansiedade.

Algumas evidências de fatores cognitivos transdiagnósticos presentes em diferentes transtornos emocionais incluem: 1) o pensamento negativo na forma de preocupação e de ruminação, envolvido na alta comorbidade entre transtornos relacionados à ansiedade (Drost et al., 2014); 2) as crenças de que as emoções são inaceitáveis e se relacionam com maior evitação emocional e com níveis mais elevados de depressão, ansiedade e fadiga (Sydenham et al., 2017); 3) $\mathrm{o}$ autocriticismo (crenças de ser falho, sem valor e inferior) e a dependência (crenças de ser frágil e indefeso) estão relacionados com ansiedade social, transtorno de estresse pós-traumático e depressão, além de transtorno da personalidade limítrofe (Kopala-Sibley, Zuroff, Hankin, \& Abela, 2015); 4) a intolerância à incerteza constitui um fator preditivo dos transtornos obsessivo-compulsivo, de ansiedade generalizada, do pânico e de ansiedade social (Shihata, McEvoy, \& Mullan, 2017), além de depressão (Payne et al., 2016); 5) a intolerância à incerteza e as metacognições negativas (percepções negativas sobre os próprios pensamentos e emoções) levam a avaliações falhas de ameaça e mediam a preocupação excessiva (McEvoy \& Mahoney, 2013). Em seu conjunto, essas evidências confirmam a necessidade de se tratar os componentes cognitivos comuns aos diversos transtornos emocionais (Clark \& Beck, 2012; Payne et al., 2016).

A elevada prevalência da ansiedade clínica no mundo e os prejuízos pessoais envolvidos têm sido um desafio para a pesquisa, prevenção e tratamento dos transtornos emocionais. Nesse sentido, várias medidas de avaliação da ansiedade foram desenvolvidas, especialmente as de autorrelato (Clark \& Beck, 2012). Algumas dessas medidas avaliam aspectos fisiológicos, em vez de cognitivos (por ex., Beck Anxiety Inventory BAI - Beck \& Steer, 1990). Outras, embora focalizem sintomas cognitivos, são relacionadas a algum tipo específico de transtorno de ansiedade e/ou o construto enquanto traço ou estado (por ex., Penn State Worry Questionnaire - PSWQ - Meyer, Miller, Metzger, \& Borkovec, 1990; State Trait Anxiety Inventory - STAI Spielberger, Gorsuch, Lushene, Vagg, \& Jacobs, 1983; Social Phobia Inventory - SPIN - Connor et al., 2000; Panic Belief Inventory - PBI - Wenzel, Sharp, Brown, Greenberg, \& Beck, 2006; Meta-Cognitions Questionaire - MCQ - Cartwright-Hatton \& Well, 1997). Nenhuma das medidas anteriormente citadas avalia, em específico, os aspectos cognitivos comuns aos transtornos de ansiedade, embora esta seja uma demanda já apontada na literatura, conforme citado anteriormente. Logo, as medidas citadas avaliam aspectos fisiológicos e, mesmo aquelas que avaliam apenas descritores 
cognitivos, o fazem focando um transtorno específico (ex., fobia social, pânico) ou mesmo o objetivo é avaliar traço-estado, o que diferencia a ECOGA de todas as medidas relatadas.

Diante do exposto, o presente estudo buscou investigar as propriedades psicométricas da Escala Cognitiva de Ansiedade (ECOGA), buscando averiguar evidências de validade de estrutura interna e confiabilidade em uma amostra formada por população geral, pacientes de instituições de saúde e amostra clínica com hipótese diagnóstica de transtorno de ansiedade. O processo completo de construção dos itens, validade de conteúdo e arcabouço teórico da ECOGA se encontra em Falcone et al. (2016).

\section{Método}

\section{Participantes}

A amostra foi selecionada por conveniência, composta por 874 participantes, sendo $656(75,1 \%)$ da população geral, 179 (20,5\%) de instituições de saúde e $39(4,5 \%)$ de pacientes de consultórios particulares com hipótese diagnóstica de transtornos de ansiedade. A amostra foi proveniente de diversos estados do Brasil, como Minas Gerais (457 - 52\%), Rio de Janeiro (316 - 36\%) e São Paulo (101 - 12\%). A média de idade foi de $30,71(D P=11,7)$ anos. O sexo masculino representou $55 \%(n=481)$ da amostra e a maioria é solteiro $(61,1 \%)$ e casado $(33,6 \%)$, além de ter ensino superior incompleto $(46 \%)$ e completo $(36,8 \%)$.

\section{Instrumento}

Foi utilizada a Escala Cognitiva de Ansiedade (ECOGA) (Falcone et al., 2016). Esta era constituída, inicialmente, por 73 itens, com formato de resposta Likert de cinco pontos (discordo a concordo completamente), baseados na Teoria Cognitiva da Ansiedade (Clark \& Beck, 2012). Inicialmente foram construídos 73 itens baseados em 17 descritores da literatura especializada em Terapia Cognitiva a respeito de sintomas de ansiedade, por intermédio de revisão narrativa da literatura nas bases de dados LILACS (Literatura Latino-Americana e do Caribe de Informação em Ciências da Saúde) e SciELO (Scientific Eletronic Library Online). Utilizou-se os booleanos: "Ansiedade e Crenças (Anxiety and Beliefs)" e "Ansiedade e Escala (Anxiety and Scale)" contidos no descritor de assunto, palavras do título ou resumo, entre os anos de 2009 a 2014. Além dos descritores decorrentes da análise de literatura também foi utilizado o livro de Clark e Beck (2012), pelo seu reconhecimento na área e por apresentar, de forma detalhada, os vieses cognitivos presentes na ansiedade clínica, além de citar muitos exemplos de pensamentos contendo esses vieses. Como resultado da análise da literatura, foram identificados 17 descritores cognitivos, organizados em quatro categorias, com base em seus conteúdos teóricos comuns (Falcone et al., 2016). Tais categorias incluíram: a) visão distorcida do perigo (tendência a antecipar, a superestimar, a ficar alerta e a selecionar pistas de ameaça, com prejuízo do raciocínio realista); b) visão distorcida dos recursos ou competências pessoais (percepção distorcida de estar vulnerável e incapaz de enfrentar a situação temida); c) catastrofização, negativismo e superestimativa da probabilidade (visão distorcida e negativa quanto à intensidade, às consequências e à probabilidade de ocorrência do perigo) e d) preocupação, evitação cognitiva ativa e passiva (tendência a evitar pensamentos ou emoções negativas, assim como imaginar estratégias antecipatórias para se pôr a salvo).

\section{Procedimentos}

O instrumento foi aplicado de forma individual (em instituições de saúde e consultórios particulares), na qual os participantes levaram o questionário para responder em casa, apenas no caso dos pacientes, justamente para não ocupar o horário da consulta. $\mathrm{Na}$ forma coletiva (em salas de aula), a coleta teve duração aproximada de 25 minutos em cada sala. Todos assinaram previamente o Termo de Consentimento Livre e Esclarecido - TCLE.

Todos os procedimentos éticos foram seguidos e estão em conformidade com a Resolução 510/2016 e em seus complementares do Conselho Nacional de Saúde. O projeto foi aprovado pelo Comitê de Ética em Pesquisa da Universidade Estadual do Rio de janeiro UERJ (Parecer 929.398).

\section{Análise de Dados}

Todas as análises foram conduzidas com o programa Factor 10.8.02 (Lorenzo-Seva \& Ferrando, 2006; Ferrando \& Lorenzo-Seva, 2017). Inicialmente foram utilizadas as análises de dimensionalidade (Análise paralela, métodos Hull e MAP) e, na sequência, foi utilizada a análise fatorial exploratória (AFE), método Minimum Rank Factor Analysis (MRFA) (Ten Berge \& Kiers, 1991) e rotação Promim para determinar quais itens iriam permanecer na versão final. Coeficientes de fidedignidade também foram calculados, além dos índices de ajuste. Os índices de confiabilidade foram expressos 
pelo Orion, para cada um dos fatores e pelo Cronbach para a escala geral.

\section{Resultados}

Inicialmente, a dimensionalidade dos itens foi investigada mediante o método Parallel Analysis - PA (Timmerman \& Lorenzo-Seva, 2011), método Hull (Lorenzo-Seva, Timmerman, \& Kiers, 2011), e Minimum Average Partial - MAP (Velicer, 1976), que apontam o número de fatores que resulta nas menores correlações parciais residuais entre os itens. No caso, a análise paralela demonstrou a existência de quatro fatores, o MAP, três fatores e o método Hull, um fator. A partir dessas informações, foram rodadas análises com três e quatro fatores, cada uma com cargas fatoriais mínimas de 0,40 e 0,50 , a fim de se optar pela melhor solução psicométrica e teórica. O método utilizado foi o Minimum Rank Factor Analysis (MRFA), com rotação Promim, para dados policóricos, método e rotação adequados para o tipo de dado (Costello \& Osborne, 2005; Damásio, 2012; Lorenzo-Seva, 1999). As análises levando-se em consideração quatro e três fatores se deram devido a alguns pontos relevantes. Em primeiro lugar, a partir de revisão teórica da literatura específica da área, quatro fatores teóricos foram propostos (Falcone et al, 2016), o que coincidiu com a análise paralela. No entanto, o método MAP apontou três fatores a partir dos dados. Apesar do método Hull ter dado a opção de unidimensionalidade, este é bastante conservador e os índices de unifatorialidade do programa Factor não confirmaram essa opção (Lorenzo-Seva et al., 2011).

A versão final de três fatores, com cargas fatoriais mínimas de 0,50 , foi a estrutura eleita, levando-se em conta a adequação dos índices de ajustes e a concatenação teórica. Os resultados indicaram índice de Kaiser-Meyer-Olkin $(\mathrm{KMO})=0,93$ e índice de Bartlett's $=6646.5(d f=465 ; P=0,000010)$, sendo considerados muito bons para o uso de procedimentos de fatoralidade. A partir dessa informação, foram efetuadas quatro rodadas de análises, sempre retirando os itens que não carregavam minimamente com 0,50 em algum dos fatores, restando 31 itens na versão final. Os eingevalues dos três fatores, bem como a variância explicada e a confiabilidade (índice Órion) foram: Fator 1- Negativismo/Pessimismo (NP) (12 itens) de 11,44 (36,9\%, 0,94); Fator 2 - Intolerância à vulnerabilidade (IV) (12 itens) de 3,1 (10\%, 0,91); Fator 3 - Intolerância à

Tabela 1.

\begin{tabular}{|c|c|c|c|c|c|c|c|c|c|c|c|}
\hline & 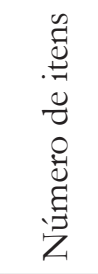 & 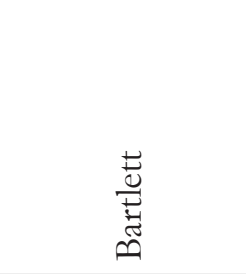 & $\stackrel{0}{\underbrace{}_{1}}$ & 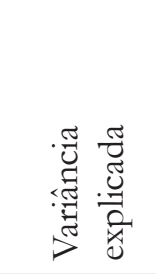 & 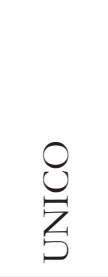 & 是 & $\overrightarrow{\mathrm{C}}$ & $\overrightarrow{\mathrm{T}}$ & $\sum_{\substack{I\\
}}^{\infty}$ & $\begin{array}{l}Z \\
0 \\
0 \\
\text { ำ } \\
0\end{array}$ & 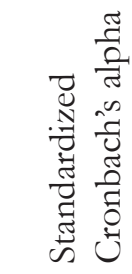 \\
\hline $\begin{array}{c}3 \text { fatores } \\
-0,40\end{array}$ & $\begin{array}{c}45 \\
\text { itens }\end{array}$ & $\begin{array}{c}10247.9 \\
(d f=990 \\
P=0.000010)\end{array}$ & $\begin{array}{c}0.94686 \\
\text { (very good) }\end{array}$ & $50,57 \%$ & 0.943 & 0.227 & 0.996 & 0.987 & 0.024 & $\begin{array}{l}0.95 \\
0.93 \\
0.93 \\
\end{array}$ & 0.96 \\
\hline $\begin{array}{c}3 \text { fatores } \\
-0,50\end{array}$ & $\begin{array}{c}31 \\
\text { itens }\end{array}$ & $\begin{array}{c}6646.5 \\
(d f=465 ; \\
P=0.000010)\end{array}$ & $\begin{array}{c}0.93188 \\
\text { (very good) }\end{array}$ & $54,62 \%$ & 0.915 & 0.272 & 0.994 & 0.988 & 0.032 & $\begin{array}{l}0.94 \\
0.91 \\
0.89\end{array}$ & 0.94 \\
\hline $\begin{array}{l}4 \text { fatores } \\
-0,40\end{array}$ & $\begin{array}{l}44 \\
\text { itens }\end{array}$ & $\begin{array}{l}10306.5 \\
(d f=946 ; P= \\
0.000010)\end{array}$ & $\begin{array}{l}0.95492 \\
\text { (very good) }\end{array}$ & $55,69 \%$ & 0.937 & 0.200 & 0.999 & 0.992 & 0.010 & $\begin{array}{l}0.88 \\
0.91 \\
0.95 \\
0.95\end{array}$ & 0.96 \\
\hline $\begin{array}{l}4 \text { fatores } \\
-0,50\end{array}$ & $\begin{array}{l}34 \\
\text { itens }\end{array}$ & $\begin{array}{l}7580.8 \\
(d f=561 ; P= \\
0.000010)\end{array}$ & $\begin{array}{l}0.94066 \\
\text { (very good) }\end{array}$ & $58,81 \%$ & 0.934 & 0.217 & 1.000 & 0.993 & 0.007 & $\begin{array}{l}0.94 \\
0.89 \\
0.94 \\
0.83\end{array}$ & 0.95 \\
\hline
\end{tabular}


incerteza (IN) (7 itens) de 2,36 (7,5\%, 0,89). A configuração final dos 31 itens restantes se encontra na Tabela 2 contendo todas as cargas fatoriais e, na Tabela 3, é possível observar somente os itens que carregaram em cada fator, com cargas acima de 0,50 .

$\mathrm{Na}$ tabela 2, é apresentada a distribuição de itens da ECOGA e as comunalidades, levando-se em consideração a solução com três fatores, com a numeração original dos 73 itens. Todas as cargas fatoriais dos itens em todos os fatores são expostas nessa tabela, diferentemente da Tabela 3 , em que somente as cargas do item em seu próprio fator é demarcado e a numeração foi redefinida para a sequência dos itens que compuseram a versão final da ECOGA.

Em relação à correlação entre os fatores, o F1 com o F2 foi de 0,58; o F1 com o F3 de 0,51 e, por fim, o F2

Tabela 2.

Análise Fatorial Exploratória (Minimum Rank Factor Analysis) Relacionando Itens e Fatores da ECOGA

\begin{tabular}{|c|c|c|c|c|}
\hline Itens & Fator 1 & Fator 2 & fator 3 & Comunalidades \\
\hline 1 & 0.115 & 0.621 & -0.052 & 0.454 \\
\hline 2 & 0.712 & 0.096 & -0.120 & 0.514 \\
\hline 8 & 0.547 & 0.198 & -0.011 & 0.456 \\
\hline 9 & -0.004 & -0.006 & 0.711 & 0.500 \\
\hline 14 & -0.044 & 0.188 & 0.692 & 0.573 \\
\hline 16 & 0.022 & 0.665 & 0.060 & 0.495 \\
\hline 17 & 0.231 & 0.533 & -0.009 & 0.475 \\
\hline 18 & 0.816 & 0.007 & -0.063 & 0.623 \\
\hline 22 & -0.137 & -0.001 & 0.784 & 0.523 \\
\hline 23 & 0.098 & 0.600 & -0.007 & 0.434 \\
\hline 24 & 0.368 & -0.211 & 0.546 & 0.508 \\
\hline 28 & 0.630 & 0.242 & -0.174 & 0.517 \\
\hline 29 & 0.827 & -0.247 & 0.146 & 0.627 \\
\hline 31 & -0.270 & 0.536 & -0.001 & 0.193 \\
\hline 34 & 0.056 & 0.749 & -0.014 & 0.604 \\
\hline 35 & 0.599 & 0.168 & 0.004 & 0.506 \\
\hline 36 & 0.021 & 0.105 & 0.542 & 0.361 \\
\hline 39 & 0.006 & 0.596 & -0.165 & 0.312 \\
\hline 43 & 0.656 & -0.133 & 0.178 & 0.481 \\
\hline 45 & -0.249 & 0.527 & 0.264 & 0.295 \\
\hline 48 & 0.092 & 0.720 & 0.009 & 0.609 \\
\hline 49 & 0.642 & 0.154 & 0.010 & 0.559 \\
\hline 51 & -0.063 & 0.093 & 0.688 & 0.483 \\
\hline 54 & 0.519 & 0.296 & -0.080 & 0.480 \\
\hline 56 & 0.849 & -0.062 & -0.030 & 0.639 \\
\hline 60 & 0.052 & 0.753 & -0.002 & 0.614 \\
\hline 61 & 0.045 & 0.117 & 0.717 & 0.633 \\
\hline 64 & 0.535 & -0.167 & 0.431 & 0.579 \\
\hline 66 & -0.049 & 0.505 & 0.048 & 0.247 \\
\hline 70 & 0.074 & 0.522 & 0.121 & 0.394 \\
\hline 73 & 0.865 & -0.118 & 0.158 & 0.796 \\
\hline
\end{tabular}


Tabela 3.

Configuração Final dos Itens (com Cargas acima de 0,50) por fator*

\begin{tabular}{|c|c|c|c|}
\hline Itens & $\begin{array}{c}\text { Fator } \\
1\end{array}$ & $\begin{array}{c}\text { Fator } \\
2\end{array}$ & $\begin{array}{c}\text { Fator } \\
3\end{array}$ \\
\hline 1- É difícil pensar em soluções quando estou nervoso(a). & & 0.621 & \\
\hline 2- Coisas ruins acontecem mais comigo do que com os outros. & 0.712 & & \\
\hline 3- Sou incapaz de resolver os problemas do dia a dia. & 0.547 & & \\
\hline 4- Devo sempre prever as situações para não ser pego(a) de surpresa. & & & 0.711 \\
\hline 5- Preciso ter o controle da situação se quiser evitar algum perigo. & & & 0.692 \\
\hline 6- O sentimento de ansiedade ou angústia pode me levar a perder o controle. & & 0.665 & \\
\hline 7- Sinto que meu medo impede que solucione meus problemas. & & 0.533 & \\
\hline 8- As coisas sempre dão errado. & 0.816 & & \\
\hline 9- Tento prever possíveis perigos ao planejar uma atividade. & & & 0.784 \\
\hline 10- Fico frágil quando não tenho alguém para me dar segurança em situações negativas. & & 0.600 & \\
\hline 11- É melhor pensar em tudo o que pode dar errado. & & & 0.546 \\
\hline 12- Tenho poucas habilidades para enfrentar os problemas da vida. & 0.630 & & \\
\hline 13- É melhor esperar que o pior aconteça. & 0.827 & & \\
\hline 14- Evito pensar em coisas ruins para não sofrer. & & 0.536 & \\
\hline 15- Quando estou nervoso(a) tenho dificuldades para raciocinar. & & 0.749 & \\
\hline 16- Quando uma coisa fora do planejado acontece, nada mais pode dar certo. & 0.599 & & \\
\hline 17- As pessoas que ficam atentas ao perigo são mais prevenidas. & & & 0.542 \\
\hline 18- Diante de alguma ameaça, preciso contar com a ajuda de alguém. & & 0.596 & \\
\hline 19- Costumo achar que as situações podem terminar da pior forma possível & 0.656 & & \\
\hline 20- Pensar em coisas ruins me faz sentir que estou em perigo e me deixa nervoso(a). & & 0.527 & \\
\hline 21- Quando fico nervoso(a), as coisas embaralham na minha cabeça & & 0.720 & \\
\hline 22- As coisas de que tenho medo sempre acontecem. & 0.642 & & \\
\hline 23- Estou sempre em alerta para as situações que possam oferecer alguma ameaça. & & & 0.688 \\
\hline 24- As pessoas são mais capazes do que eu de enfrentar situações ameaçadoras. & 0.519 & & \\
\hline 25- Quando alguma coisa pode dar errado, com certeza vai dar. & 0.849 & & \\
\hline 26- Quando estou preocupado(a) ou ansioso(a) não consigo pensar com clareza. & & 0.753 & \\
\hline 27- Preciso estar alerta para evitar que coisas ruins aconteçam. & & & 0.717 \\
\hline 28- Pensar no pior é uma maneira de não ser pego(a) de surpresa. & 0.535 & & \\
\hline 29- É melhor evitar falar/pensar sobre assuntos desagradáveis que geram angústia. & & 0.505 & \\
\hline 30- É insuportável me sentir ansioso(a). & & 0.522 & \\
\hline 31- Geralmente espero que o pior aconteça. & 0.865 & & \\
\hline
\end{tabular}

O Fator 1, denominado de negativismo/pessimismo (N.P.), ficou constituído pelos itens: 2, 3, 8, 12, 13, 16, 19, 22, 24, 25, 28 e 31; o Fator 2, intolerância à vulnerabilidade (I.V.) contém os itens: 1, 6, 7, 10, 14, 15, 18, 20, 21, 26, 29, e 30 e, por fim, o Fator 3, intolerância à incerteza (IN), agrupou os itens $4,5,9,11,17,23$ e 27.

com o F3 de 0,37 . Os índices de ajuste do modelo escolhido com três fatores foram: Comparative Fit Index (CFI) foi de 0,994; o Goodness of Fit Index (GFI) de 0,988, e o Root Mean Square Error of Approximation (RMSEA) atingiu o valor de 0,032 .

\section{Discussão}

O objetivo deste estudo foi avaliar a estrutura interna de uma nova medida denominada Escala Cognitiva de Ansiedade, baseada nos descritores cognitivos 
de ansiedade, de acordo com uma perspectiva transdiagnóstica, que tem como objetivo identificar fatores cognitivos comuns aos transtornos emocionais (Barlow \& Durand, 2017; Franzin et al., 2018; Payne et al., 2016). Nesse sentido, uma medida que possa ser utilizada, independentemente do transtorno de ansiedade específico, tende a ser mais apropriada no rastreamento, bem como no acompanhamento de intervenções clínicas. Importante lembrar que este é o primeiro estudo de evidências de validade baseada na estrutura interna, a partir de análise fatorial exploratória (AFE). No estudo de Falcone et al. (2016), houve a construção e evidências de validade baseada no conteúdo da ECOGA, com a proposição de quatro fatores teóricos baseados na literatura, que foram testados em comparação a uma versão com menor número de itens e o agrupamento de três fatores também coerentes com a literatura (Clark \& Beck, 2012).

O objetivo principal da análise de estrutura interna foi a de obter uma escala com o menor número possível de itens, ou seja, aqueles com maiores cargas fatoriais, com índices de ajustes adequados e coerente com uma vasta gama de literatura em aspectos cognitivos da ansiedade. Sendo assim, ao se comparar os modelos de três e quatro fatores, aquele com 31 itens, três fatores e cargas acima de 0,50 foi o predileto, mesmo que todos os outros modelos possuíssem índices também adequados. A predileção por esse modelo foi principalmente pelo número menor de itens e pela adaptação teórica cognitiva na visão transdiagnóstica. Assim, os índices de ajustes, como o CFI e GFI foram sempre maiores que 0,95 e o RMSEA abaixo de 0,05 (Yuan, Chan, Marcoulides, \& Bentler, 2016; Tanaka, 1993; Thompson, 2004; Yu \& Muthen, 2002). Em relação aos índices de proposição de unidimensionalidade, tem-se Unidimensional Congruence (UniCo) e o Mean of Item Residual Absolute Loadings (MIREAL) também apontando a não unifatoralidade da ECOGA, o que dispensaria a solução de Hull, sempre mais conservadora (Lorenzo-Seva et al., 2011). A variância explicada do modelo foi próxima a 55\%, bastante adequada para construtos das ciências sociais (Damásio, 2012). Cabe ressaltar que os índices de confiabilidade foram bastante adequados, tanto nos fatores isolados (mínimo de $0,89)$ quanto na escala geral $(0,94)$.

As análises fatoriais exploratórias da ECOGA indicam os bons resultados psicométricos referentes a sua estrutura interna. Os fatores descritos são congruentes com o construto da ansiedade e de seus descritores cognitivos envolvidos, identificados no estudo de validade de conteúdo (Falcone et al., 2016), bem como estão de acordo com o modelo transdiagnóstico na ansiedade (Barlow \& Durand, 2017; Franzin et al., 2018, Payne et al., 2016).

Indivíduos com escores elevados no fator Negativismo/Pessimismo (NI) tendem a fazer avaliações catastróficas/pessimistas sobre si e sobre os acontecimentos, considerando as situações como mais ameaçadoras/negativas do que realmente são e a si mesmos como incapazes de enfrentá-las, de acordo com o pior cenário possível (Clark \& Beck, 2012). É o que costuma ser referido como "fazer tempestade em um copo d'água". Já os indivíduos com escores elevados no fator Intolerância à vulnerabilidade (IV) costumam considerar que estar vulnerável significa ser fraco, frágil ou incapaz de funcionar eficazmente. Desse modo, tendem a regular a emoção evitando falar ou pensar em coisas que podem deixá-los ansiosos, além de usarem a distração e o distanciamento emocional. A busca de segurança por meio de reasseguramento pode também ser utilizada diante da percepção de estar vulnerável. O senso aumentado de vulnerabilidade é identificado na intolerância à ansiedade (Sydenham et al., 2017), aumentando a percepção de impotência frente a situação considerada como perigosa, predispondo à evitação cognitiva e emocional (Payne et al., 2016).

Por fim, indivíduos com escores elevados no fator Intolerância à incerteza (IN) superestimam a probabilidade de acontecimentos ameaçadores e costumam considerar as incertezas da vida como insuportáveis. Uma vez que a maior parte dos acontecimentos não são previsíveis, eles se engajam em episódios de preocupação/ruminação como forma ineficaz de prevenir o perigo e de obter alívio. As preocupações se constituem em elaborações mentais que visam encontrar saídas para prevenir consequências negativas. A intolerância à incerteza parece estar na base das preocupações (McEvoy \& Mahoney, 2013). A preocupação resulta em maior reavaliação da ameaça e da ansiedade subjetiva, uma vez que gera um estado de apreensão ansiosa (Clark \& Beck, 2012; McEvoy \& Mahoney, 2013). A incapacidade para tolerar situações ambíguas do ambiente leva à hipervigilância, entendida como foco excessivo da atenção frente a situação considerada como perigosa (interna ou externa), com o objetivo de obter controle sobre a situação. Tanto a hipervigilância quanto o controle contribuem para a elevação da ansiedade (Clark \& Beck, 2012) e são identificadas em indivíduos com escores elevados nesse fator. 
As dimensões do construto identificadas no primeiro estudo: a) visão distorcida do perigo; b) visão distorcida dos recursos pessoais; c) catastrofização, negativismo e superestimação da probabilidade e d) preocupação, evitação cognitiva ativa e passiva representam as quatro categorias construídas a partir dos 17 descritores cognitivos específicos da ansiedade, identificados em cada item do ECOGA, no estudo de validade de conteúdo (Falcone et al., 2016). Após a análise fatorial, esses descritores foram reorganizados de acordo com os itens agrupados nos três Fatores.

Os itens que compõem o Fator 1 (Negativismo/ Pessimismo), reuniram itens com os seguintes descritores: cognições catastróficas; visão pessimista/previsões negativas; percepção pessoal negativa; superestimação do perigo e percepção de vulnerabilidade. Já o Fator 2 (Intolerância a vulnerabilidade) reuniu: intolerância a afetos negativos; prejuízo do pensamento racional; controle/evitação dos pensamentos e busca de ajuda/ segurança. Finalmente, os descritores identificados nos itens do Fator 3 (Intolerância à incerteza) foram: intolerância à imprevisibilidade/incerteza; importância de controlar os pensamentos; preocupação excessiva/ ruminação; e hipervigilância.

Os três fatores identificados a partir da análise fatorial são congruentes com os 13 dos 17 descritores cognitivos identificados no primeiro estudo de validação. Além disso, eles estão também relacionados a alguns dos fatores transdiagnósticos apontados anteriormente, como preocupação e ruminação (Drost et al., 2014); evitação emocional (Sydenham et al., 2017); crenças de ser frágil e indefeso (Kopala-Sibley et al., 2015); intolerância à incerteza e cognições pessoais negativas ou autocriticismo (Shihata et al., 2017).

A ECOGA é uma escala brasileira, baseada no modelo cognitivo de Beck (Clark \& Beck, 2012), cujos fatores confirmam vários vieses cognitivos relativos ao processamento cognitivo característico da ansiedade, independentemente dos critérios diagnósticos preenchidos pelo respondente, baseados na visão mais recente da Terapia Cognitiva (Beck \& Haigh, 2014). Suas subescalas também identificam fatores transdiagnósticos apontados na literatura (Payne et al., 2016), tal como citados nos estudos sobre intolerância às emoções (Sydenham et al., 2017); intolerância à incerteza (McEvoy \& Mahoney, 2013; Shihata et al., 2017); metacognições negativas e preocupação (McEvoy \& Mahoney, 2013). Desse modo, a ECOGA pode ser uma escala útil para a realização de pesquisas que avaliem fatores cognitivos de ansiedade, presentes em diferentes transtornos psicológicos. Pode também ser utilizada em conjunto com medidas que avaliam algum tipo específico de transtorno de ansiedade, como fobia social ou transtorno do pânico (Connor et al., 2000; Wenzel et al., 2006).

Uma vez que a intolerância à incerteza e à vulnerabilidade, assim como padrões de pensamentos negativistas estão presentes na maioria dos transtornos de ansiedade e de humor, estes podem ser o principal foco de tratamento em indivíduos vulneráveis a tais padrões e que apresentam transtornos comórbidos (Payne et al., 2016). Assim, a ECOGA poderá contribuir fortemente, não somente para a avaliação desses padrões cognitivos, mas também para o seu tratamento. As medidas de ansiedade também auxiliam na avaliação clínica por meio de seu uso repetido durante o processo de tratamento, para verificação da redução dos sintomas cognitivos e, portanto, dos efeitos do tratamento (Falcone et al., 2016). Essas constatações reafirmam a utilidade da ECOGA para avaliar e tratar os sintomas cognitivos presentes nos clientes de psicoterapia ou de ambulatórios. Considerando-se que a literatura tem incentivado a realização de pesquisas que identifiquem o papel de cognições distorcidas comuns aos transtornos de ansiedade (Clark \& Beck, 2012), a ECOGA poderá contribuir para identificar ou confirmar estudos que avaliam as relações entre as suas três subescalas e esses transtornos.

Um dos pontos que devem ser levados em consideração foi que somente a amostra clínica respondeu ao instrumento em casa. Apesar de ser um número pequeno do total de todos os respondentes (4,5\%), não foi possível haver tal padronização, sendo uma limitação do tipo de amostra e locais onde foram realizadas as coletas. Estudos futuros podem ser desenvolvidos no sentido de propor, em novas amostras, análises confirmatórias do modelo encontrado, bem como evidência de validade baseada na relação com variáveis externas. Além dessas evidências de validade, com o aumento da amostra clínica, também será possível realizar estudos de acurácia diagnóstica, especificamente, a partir da curva ROC, calcular os índices de sensibilidade e especificidade, tão importantes para escalas que avaliam características psicopatológicas, além de outros estudos com a ECOGA, ampliando assim estudos de novas evidências de validade, como apontado nos Standards (American Educational Research Association [AERA], American Psychological Association [APA], National Council on Measurement in Education [NCME], 2014); Runeson et al., 2017). Pesquisas futuras utilizando 
amostras representativas, além de outros grupos clínicos também devem ser implementadas a fim de avaliar se a estrutura da ECOGA se mantém estável.

\section{Referências}

American Educational Research Association, American Psychological Association, \& National Council on Measurement in Education (2014). Standards for educational and psychological testing. Washington, DC: American Psychological Association.

Barlow, D. H., \& Durand, V. M. (2017). Psicopatologia. Uma abordagem integrada (2 $2^{\mathrm{a}}$ ed.). São Paulo: Centage Learning.

Beck, A. T., \& Haigh, E. A. P. (2014). Advances in Cognitive Theory and Therapy: The Generic Cognitive Model. Annual Review of Clinical Psychology, 10, 1-24. doi: 10.1146/annurev-clinpsy-032813-153734

Beck, A. T., \& Steer, R. A. (1990). Manual for the Beck Anxiety Inventory. San Antonio: Psychological Corporation.

Blay, S. L., Fillenbaumb, G. G., Mello, M. F., Quintanaa, M. I., Mari, J. J., Bressana, R. A., \& Andreolia, S. B. (2018). 12-month prevalence and concomitants of DSM-IV depression and anxiety disorders in two violence-prone cities in Brazil. Journal of Affective Disorders, 232, 204-211. doi: 10.1016/j. jad.2018.02.023

Cartwright-Hatton, S., \& Wells, A. (1997). Beliefs about worry and intrusions: The meta-cognitions questionnaire and its correlates. Journal of anxiety disorders, 11(3), 279-296. doi: 10.1016/ S0887-6185(97)00011-X

Clark, D. A., \& Beck, A. T. (2012). Terapia cognitiva para os transtornos de ansiedade. Porto Alegre: Artmed.

Connor, K. M., Davidson, J. R. T., Churchill, L. E., Sherwood, A., Foa, E., \& Weisler, R. H. (2000). Psychometric properties of the Social Phobia Inventory (SPIN): New self-rating scale. The British Journal of Psychiatry, 176(4), 379-386. doi: 10.1192/ bjp.176.4.379

Costello, A. B., \& Osborne, J. W. (2005). Best practices in exploratory factor analysis: Four recommendations for getting the most from your analysis. Pratical Assessment, Research \& Evaluation, 10(7),
1-9. Recuperado de http://pareonline.net/getvn. asp?v $=10 \& n=7$

Damásio, B. F. (2012). Uso da análise fatorial exploratória em Psicologia. Avaliação Psicológica, 11(2), 213-228. Recuperado de http://pepsic.bvsalud. org/pdf/avp/v11n2/v11n2a07.pdf

Drost, J., van der Does, W., van Hemert, A. M., Pennix, B. W., \& Spinhoven, P. (2014). Repetitive negative thinking as a transdiagnostic factor in depression and anxiety: A conceptual replication. Behaviour Research and Therapy, 63, 177-183. doi:10.1016/j. brat.2014.06.004

Falcone, E. M. O., Baptista, M. N., Placido, M. G., Krieger, S., Oliveira, E. R., Falcone, J. F., \& Vieira, B. F. L. (2016). Construção e validade de conteúdo da Escala Cognitiva de Ansiedade em adultos. Psicologia em Pesquisa, 10, 85-93. doi: $10.24879 / 201600100010050$

Ferrando, P. J., \& Lorenzo-Seva, U. (2017). Program FACTOR at 10: Origins, development and future directions. Psicothema, 29(2), 236-240. doi: 10.7334/ psicothema2016.304

Franzin, R., Caetano, K. A. S., \& Melo, W. V. (2018). Tratamentos transdiagnósticos eficazes em terapia cognitivo-comportamental. Em Federação Brasileira de Terapias Cognitivas, C. B. Neufeld, E. M. O. Falcone, \& B. P. Rangé. (Eds.). PROCOGNITIVA Programa de Atualização em Terapia Cognitivo-Comportamental: Ciclo 4. (pp. 31-37). Porto Alegre: Artmed Panamericana. (Sistema de Educação Continuada a Distância, v. 4).

GBD 2016 DALYs and HALE Collaborators. (2017). Global, regional, and national disability-adjusted life-years (DALYs) for 333 diseases and injuries and healthy life expectancy (HALE) for 195 countries and territories, 1990-2016: A systematic analysis for the Global Burden of Disease Study 2016. Lancet, 390(10100), 1260-1334. doi: 10.1016/ S0140-6736(17)32130-X

Kopala-Sibley, D. C., Zuroff, D. C., Hankin, B. L., \& Abela, J. R. (2015). The development of self-criticism and dependency in early adolescence and their role in the development of depressive and anxiety symptoms. Personality and Social Psychology Bulletin, 41(8), 1094-1109. doi: 10.1177/0146167215590985 
Lorenzo-Seva, U. (1999). Promin: a method for oblique factor rotation. Multivariate Behavioral Research, 34(3), 347-365. doi: 10.1207/S15327906MBR3403_3

Lorenzo-Seva, U., \& Ferrando, P. J. (2006). FACTOR: A computer program to fit the exploratory factor analysis model. Behavior Research Methods, 38(1), 8891. doi:10.3758/BF03192753

Lorenzo-Seva, U., Timmerman, M. E., \& Kiers, H. A. L. (2011). The Hull method for selecting the number of common factors. Multivariate Behavioral Research, 46(2), 340-364. doi: 10.1080/00273171.2011.564527

McEvoy, P. M., \& Mahoney, A. E. J. (2013). Intolerance of uncertainty and negative metacognitive beliefs as transdiagnostics mediators of repetitive negative thinking in a clinical sample withanxiety disorders. Journal of Anxiety Disorders, 27(2), 216224. doi: 10.1016/j.janxdis.2013.01.007

Meyer, T. J., Miller, M. L., Metzger, R. L., \& Borkovec, T. D. (1990). Development andvalidation of the Penn State Worry Questionnaire, Behaviour Research and Therapy, 28(6), 487-495. doi:10.1016/0005-7967(90)90135-6

Payne, L.A., Ellard, K. K., Farchione, T. J., Fairholme, C. P., \& Barlow, D. H. (2016). Transtornos emocionais. Um protocolo unificado transdiagnóstico. Em D. H. Barlow (Ed.). Manual clínico dos transtornos psicológicos: Tratamento passo a passo (5a ed., pp. 237274). Porto Alegre: Artmed.

Runeson, B., Odeberg, J., Pettersson, A., Edbom, T., Adamsson, J. I., \& Waern, M. (2017). Instruments for the assessment of suicide risk: A systematic review evaluating the certainty of the evidence. PLOS ONE, 12(7). doi: 10.1371/journal.pone.0180292

Shihata, S., McEvoy, P. M., \& Mullan, B. A. (2017). Pathways from uncertainty to anxiety: An evaluation of a hierarchical model of trait and disorder-specific intolerance of uncertainty on anxiety disorder symptoms. Journal of Anxiety Disorders, 45, 72-79. doi: 10.1016/j.janxdis.2016.12.001

Spielberger, C. D., Gorsuch, R. L., Lushene, R., Vagg, P. R., \& Jacobs, G. A. (1983). Manual for the State-Trait Anxiety Inventory. Palo Alto: Consulting Psychologists Press.
Sydenham, M., Beardwood, J., \& Rimes, K.A. (2017). Beliefs about emotions, depression, anxiety and fatigue: A mediational analysis. Behavioural and Cognitive Psychotherapy, 45(1), 73-78. doi: 10.1017/ S1352465816000199

Tanaka, J. (1993). Multifaceted conceptions of fit in structural equation models. Em K. Bollen and J. Long (Eds.). Testing structural equation models (pp. 1039). Sage Press: Newbury Park.

Ten Berge, J. M. F., \& Kiers, H. A. L. (1991). A numerical approach to the approximate and the exact minimum rank of a covariance matrix. Psychometrika, 56(2), 309-315. doi: 10.1007/BF02294464

Thompson, B. (2004). Exploratory and confirmatory factor analysis: Understanding concepts and applications. Washington: American Psychological Association. doi: 10.1037/10694-000

Timmerman, M. E., \& Lorenzo-Seva, U. (2011). Dimensionality assessment of ordered polytomous items with parallel analysis. Psychological Methods, 16(2), 209-220. doi:10.1037/a0023353

Velicer, W. F. (1976). Determining the number of components from the matrix of partial correlations. Psychometrika, 41(3), 321-327. doi: 10.1007/ BF02293557

Wenzel, A., Sharp, I. R., Brown, G. K., Greenberg, R. L., \& Beck, A. T. (2006). Dysfunctional beliefs in panic disorder: The Panic Belief Inventory.Behaviour Research and Therapy, 44(6), 819-833. doi: 10.1016/j.brat.2005.06.001

Yu, C. Y., \& Muthén, B. (2002). Evaluation of model fit indices for latent variable models with categorical and continuous outcomes. Paper presented at the annual meeting of the American Educational Research Association, New Orleans, LA.

Yuan, K. H., Chan, W., Marcoulides, G. A., \& Bentler, P. M. (2016). Assessing structural equation models by equivalence testing with adjusted fit indexes. Structural Equation Modeling: A Multidisciplinary Journal, 23(3), 319-330. doi:10.1080/10705511.2015.1 065414

Recebido em: 27/12/2018

Reformulado em: 30/10/2019

Aprovado em: 03/02/2020 
Sobre os autores:

Makilim Nunes Baptista tem Graduação em Psicologia pela Universidade São Judas Tadeu, mestrado em Psicologia pela Pontifícia Universidade Católica de Campinas e doutorado pelo departamento de Psiquiatria e Psicologia Médica da Universidade Federal de São Paulo. Docente do Programa de Pós-Graduação Stricto-Sensu em Psicologia da Universidade São Francisco-Campinas. Bolsista produtividade pelo CNPq; Coordenador do Laboratório de Avaliação Psicológica em Saúde Mental (LAPSAM-III) do Programa de Pós- Graduação Stricto-Sensu em Psicologia da Universidade São Francisco; Presidente do Instituto Brasileiro de Avaliação Psicológica - IBAP; Membro do Grupo de Trabalho de Família da União Latino-Americana de Entidades de Psicologia (ULAPSI); Membro del Red Mundial Suicidólogos.

ORCID: https://orcid.org/0000-0001-6519-254x

E-mail:makilim01@gmai.com

Eliane Mary de Oliveira Falcone é Mestre em psicologia clínica pela PUC-RIO; Doutora em psicologia clínica pela USP; Pós-doutora em psicologia clínica pela PUCRS e em psicologia experimental pela USP. Docente do PPGPS (UERJ). Especialista em Terapia Cognitiva pelo Beck Institute. Terapeuta certificada pela Federação Brasileira de Terapias Cognitivas. Ex-presidente da FBTC (gestão 2003-2005); Fundadora da Revista Brasileira de Terapias Cognitivas (RBTC)

ORCID: https://orcid.org/0000-0002-9670-8999

E-mail: elianefalcone@uol.com.br

Monique Placido Viegas é Psicóloga graduada pela Universidade do Estado do Rio de Janeiro (UERJ). Mestre e Doutoranda em Psicologia Social (UERJ). Especialista em Terapia Cognitivo-Comportamental (Cognitivo-FADISMA).

ORCID: https://orcid.org/0000-0002-7127-0425

E-mail:monique_placido@yahoo.com.br

Evlyn Rodrigues Oliveira é Psicóloga graduada pela Universidade do Estado do Rio de Janeiro (UERJ). Mestre e Doutoranda em Psicologia Social (UERJ). Especialista em Terapia Cognitivo-Comportamental (Cognitivo-FADISMA) e Psicologia Positiva (PUCRS). Docente em cursos de Pós-Graduação em TCC e Supervisora clínica (SPA-UERJ). Autora do livro "Calçando os sapatos do outro: descobrindo os caminhos da empatia".

ORCID: https://orcid.org/0000-0003-4798-2497

E-mail: oliveira.er@live.com

Stèphanie Krieger é Psicóloga (UERJ), com formação em Terapia Cognitivo-Comportamental (particular) e Terapia do Esquema (Wainer/ISST). Mestre e Doutoranda em Psicologia Social (UERJ). Docente da graduação em Psicologia e da Pós-Graduação em Terapia Cognitivo-Comportamental da Universidade Estácio de Sá.

ORCID: https://orcid.org/0000-0001-8491-8078

E-mail: stephanie.krieger@yahoo.com.br

Luisa Braga Pereira é Mestranda no Programa de Pós-Graduação em Psicologia Social pela Universidade do Estado do Rio de Janeiro (UERJ) e Psicóloga pela UERJ. Suas temáticas de pesquisa abarcam as habilidades sociais, a ansiedade, o raciocínio dedutivo, a terapia cognitivo-comportamental e os transtornos de personalidade.

ORCID: https://orcid.org/0000-0003-4474-3298

E-mail:1ubrpereira@gmail.com

Ana Júlia de Carvalho Pereira Alves é Estudante de Graduação em Psicologia na Universidade do Estado do Rio de Janeiro. Tem experiência na área de pesquisa com ênfase em Ansiedade, Terapia Cognitivo-Comportamental e Habilidades Sociais. Atualmente, é bolsista de Iniciação Científica pela UERJ e participa de dois projetos de extensão. ORCID: https://orcid.org/0000-0003-1473-43664

E-mail: anajualves@gmail.com

Psico-USF, Bragança Paulista, v. 25, n. 4, p. 751-762, out./ dez. 2020 
Marcelo Leonel Peluso é Graduando em Psicologia pela Universidade do Estado do Rio de Janeiro (UERJ) e Membro-Representante da Liga Acadêmica de Terapias Cognitivo-Comportamentais (LATCC) da UERJ. Idealizador do Encontro de Combate e Prevenção ao Suicídio (ECPS). Suas áreas de interesse são: TCC, Ansiedade, Suicídio, Habilidades Sociais e Psicometria.

ORCID: https://orcid.org/0000-0002-3669-3561

E-mail: marceloleonelpeluso@gmail.com

Filipe Tomé da Silva é Mestrando no Programa de Pós-Graduação em Psicologia Social pela Universidade do Estado do Rio de Janeiro (UERJ) e Psicólogo graduado pela UERJ. Suas temáticas de pesquisa abarcam ansiedade, terapia cognitivo-comportamental, terapia de família e adultez emergente.

ORCID: https://orcid.org/0000-0002-1446-6464

E-mail: camilats.psi@gmail.com

Contato com os autores:

E-mail:makilim01@gmai.com 